\title{
Ear microbiota and middle ear disease: a longitudinal pilot study of Aboriginal children in a remote south Australian setting
}

Steven L. Taylor ${ }^{1,2 *}$, Lito E. Papanicolas ${ }^{1,2}$, Alyson Richards', Furdosa Ababor ${ }^{1}$, Wan Xian Kang ${ }^{1}$, Jocelyn M. Choo ${ }^{1,2}$, Charmaine Woods ${ }^{2,3}$, Steve L. Wesselingh ${ }^{4}$, Eng H. Ooi ${ }^{2,3}$, Patricia MacFarlane ${ }^{3 \dagger}$ and Geraint B. Rogers ${ }^{1,2+}$

\begin{abstract}
Background: Otitis media $(\mathrm{OM})$ is a major disease burden in Australian Aboriginal children, contributing to serious long-term health outcomes. We report a pilot analysis of OM in children attending an outreach ear and hearing clinic in a remote south Australian community over a two-year period. Our study focuses on longitudinal relationships between ear canal microbiota characteristics with nasopharyngeal microbiota, and clinical and treatment variables.

Results: Middle ear health status were assessed in 19 children (aged 3 months to 8 years) presenting in remote western South Australia and medical interventions were recorded. Over the two-year study period, chronic suppurative OM was diagnosed at least once in 7 children (37\%), acute OM with perforation in 4 children (21\%), OM with effusion in 11 children (58\%), while only 1 child had no ear disease. Microbiota analysis of 19 children (51 sets of left and right ear canal swabs and nasopharyngeal swabs) revealed a core group of bacterial taxa that included Corynebacterium, Alloiococcus, Staphylococcus, Haemophilus, Turicella, Streptococcus, and Pseudomonas. Within-subject microbiota similarity (between ears) was significantly greater than inter-subject similarity, regardless of differences in ear disease $(p=0.0006)$. Longitudinal analysis revealed changes in diagnosis to be associated with more pronounced changes in microbiota characteristics, irrespective of time interval. Ear microbiota characteristics differed significantly according to diagnosis $(\mathrm{P}(\mathrm{perm})=0.0001)$. Diagnoses featuring inflammation with tympanic membrane perforation clustering separately to those in which the tympanic membrane was intact, and characterised by increased Proteobacteria, particularly Haemophilus influenzae, Moraxella catarrhalis, and Oligella. While nasopharyngeal microbiota differed significantly in composition to ear microbiota $(P(p e r m)=0.0001)$, inter-site similarity was significantly greater in subjects with perforated tympanic membranes, a relationship that was associated with the relative abundance of $H$. influenzae in ear samples $\left(r_{s}=-0.71, p=0.0003\right)$. Longitudinal changes in ear microbiology reflected changes in clinical signs and treatment.
\end{abstract}

Conclusions: Children attending the ear and hearing clinic in a remote Aboriginal community present with a broad spectrum of OM conditions and severities, consistent with other remote Aboriginal communities. Ear microbiota

\footnotetext{
*Correspondence: steven.taylor@sahmri.com

${ }^{\dagger}$ Patricia MacFarlane and Geraint B. Rogers are Co-senior authors.

${ }^{2}$ College of Medicine and Public Health, Flinders University, Adelaide,

South Australia, Australia

Full list of author information is available at the end of the article
}

(C) The Author(s) 2022. Open Access This article is licensed under a Creative Commons Attribution 4.0 International License, which permits use, sharing, adaptation, distribution and reproduction in any medium or format, as long as you give appropriate credit to the original author(s) and the source, provide a link to the Creative Commons licence, and indicate if changes were made. The images or other third party material in this article are included in the article's Creative Commons licence, unless indicated otherwise in a credit line to the material. If material is not included in the article's Creative Commons licence and your intended use is not permitted by statutory regulation or exceeds the permitted use, you will need to obtain permission directly from the copyright holder. To view a copy of this licence, visit http://creativecommons.org/licenses/by/4.0/. The Creative Commons Public Domain Dedication waiver (http://creativeco mmons.org/publicdomain/zero/1.0/) applies to the data made available in this article, unless otherwise stated in a credit line to the data. 
characteristics align with $\mathrm{OM}$ diagnosis and change with disease course. Nasopharyngeal microbiota characteristics are consistent with the contribution of acute upper respiratory infection to OM aetiology.

Keywords: Otitis media, Ear infection, Remote, Aboriginal, Indigenous, Microbiome

\section{Background}

Otitis media $(\mathrm{OM})$ is a spectrum of pathologies that involve inflammation and/or infection of the middle ear. Characterised clinically by abnormalities of the tympanic membrane and fluid in the middle ear space, OM encompasses both acute and chronic disease, and varies in clinical presentations $[1,2]$. Otitis media with effusion (OME) is common and defined as the presence of persistent fluid behind an intact eardrum, in the absence of the signs of symptoms of acute infection, while acute otitis media (AOM) involves fluid behind the eardrum with features of fever, pain, eardrum bulging, redness, or recent discharge. AOM can occur with or without perforation of the eardrum. Where persistent discharge through a perforation of the eardrum occurs for 6 weeks or more, it is defined as chronic suppurative otitis media (CSOM) [1]. All of these forms of OM are more common in childhood and are associated with increased risk of conductive hearing loss.

$\mathrm{OM}$ is highly prevalent in indigenous populations globally, occurring more frequently, with earlier onset, and in more severe forms, compared to non-indigenous populations [1, 3-5]. In Australia, Aboriginal children in some remote communities have prevalence rates of CSOM of $30-42 \%$ [1, 6], while non-Aboriginal Australian children have amongst the lowest rates in the world [6]. OM also has a significantly greater duration in Australian Aboriginal children, averaging 32 months compared with just 3 months in non-Aboriginal children [1]. This high burden of disease is compounded by a reliance on fly in-fly out specialist services in remote communities as well as a high turnover of resident primary health care professionals [7]. High incidence of OM in Aboriginal children contributes to higher rates of temporary and permanent hearing impairment [8], which is associated with reduced school attendance [9], poorer academic outcomes [10], and wellbeing [11], and increased likelihood of substance abuse [12] and contact with the criminal justice system $[1,13,14]$.

The profound impact of $\mathrm{OM}$ on the long-term health and well-being of Australian Aboriginal children demands the development of more effective prevention and treatment strategies. Specific prevention, diagnosis, and treatment guidelines for OM in Australian Aboriginal and Torres Strait Islander children have been developed in recognition of the unique burden of disease and healthcare provision challenges in remote sites [15]. Of particular consideration is the microbiology of OM. Our understanding of the significance of ear microbiota characteristics, both as a contributor to aetiology and as a disease biomarker, is limited at present. Otopathogens, such as Streptococcus pneumoniae, Haemophilus influenzae, and Moraxella catarrhalis, are commonly identified [16, 17] and are the principal targets of medical intervention through antibiotic therapy. Notably, rates of otopathogen colonisation in Indigenous children with $\mathrm{OM}$ are particularly high and CSOM appears to be associated with a different microbiological profile compared to other OM conditions [18]. Prospective cohort studies in Australian Indigenous children have also reported nasopharyngeal carriage of these principal otopathogens to be a risk factor for OM in some studies [19, 20], but not all [17]. Moreover, medical interventions including liberal antibiotic prescription and vaccination programs have had limited effectiveness in indigenous populations [21-23].

Achieving a better understanding of the relationships between ear and nasopharyngeal microbiology and OM clinical course would inform efforts to reduce incidence and improve outcomes in Aboriginal children living in remote settings. There is an increasing appreciation that otopathogens exist in a wider context of other bacteria that can influence risk and outcome of OM, both positively or negatively $[17,18,24]$. To this end, our aim was to explore OM presentation and microbiology in children attending an outreach clinic in a remote community in South Australia, including disease characteristics between and within children over a two-year period, and to relate these to characteristics of the ear canal and nasopharyngeal microbiota.

\section{Methods}

\section{Ethical approval and consent to participate}

Ethics approval for this study was obtained from the Southern Adelaide Clinical Human Research Ethics Committee (249.15) and the Aboriginal Health Research Ethics Committee (04-15-615) and all methods were carried out in accordance with relevant guidelines and regulations (declaration of Helsinki). Children in the Yalata community in South Australia were examined by a visiting specialist ear nose and throat surgeon at the Tullawon Health Clinic as part of a pre-existing outreach programme. With the assistance of nursing staff and Aboriginal Health Workers (AHW) from the Aboriginal Health Service, parents and carers of children in the community 
were informed of the visits, and parents/caregivers were encouraged to bring their children to the clinic for assessment. With the assistance of AHW, informed consent to participate in the study was obtained from parents/ guardians once the research project had been explained to them in either their local dialect (Pitjantjatjara) or English. During specialist visits to the community, children underwent a physical examination and age-appropriate hearing screening.

\section{Study setting and population overview}

The Yalata region is on Aboriginal owned land on the edge of the Nullarbor Plains, approximately $200 \mathrm{~km}$ from the nearest regional centre and $800 \mathrm{~km}$ from metropoli$\tan$ Adelaide. The community is composed of several family groups. The population in the community fluctuates as people move between surrounding communities for work, education, medical, ceremonial, and familial reasons. At the 2016 national census, there were approximately 200 Aboriginal people living in the community, one-quarter of whom where under the age of 14 . There is a Community Controlled Health Service, staffed by full time Aboriginal Health Works, Remote Area Nurses and with regular visiting general practitioner, specialist, and allied health services.

Participants were 19 children aged 3 months to 7 years at time of enrolment, with longitudinal samples collected from 15 participants. Samples were collected over a 2-year period (Nov 2015-Nov 2017). Participant demographics and sample collection information are described in Table 1 and Fig. 1. Vaccination records were available for $14 / 19$ participants, with all 14 having vaccinations that were up to date with their age group.

\section{Sample collection}

Anonymised bilateral ear and nasopharyngeal swabs were taken from those children enrolled in the study, who were present in the community at the time of each outreach visits. Ear canal swab collection consisted of Copan flocked swabs (FLOQSwabs ${ }^{\circledR}$ 553C) inserted into the ear canal as close to the tympanic membrane as the child would permit. Nasopharyngeal swab collection consisted

Table 1 Participant demographics and clinical information during study period

\begin{tabular}{|c|c|}
\hline Variable & Number \\
\hline Number of participants & 19 \\
\hline Age at first visit, median years (min, max) & $3.20(0.23,8.75)$ \\
\hline Female, n (\%) & $10(53 \%)$ \\
\hline Aboriginal or Torres Strait Islander, n (\%) & $19(100 \%)$ \\
\hline \multicolumn{2}{|l|}{ Vaccination status, n (\%) } \\
\hline Up to date & $14(74 \%)$ \\
\hline Unknown & $5(26 \%)$ \\
\hline Number of participants with > 1 timepoint, n (\%) & $14(74 \%)$ \\
\hline Number of visits with swabs collected, median (min, max) & $2(1,7)$ \\
\hline Days between first and last swab (in those with $>1$ timepoint), median (min, max) & $485(150,730)$ \\
\hline \multicolumn{2}{|l|}{ Participants with OM during study period, n (\%) } \\
\hline CSOM & $7(37 \%)$ \\
\hline AOMwP & $4(21 \%)$ \\
\hline $\mathrm{AOM}$ & $1(5 \%)$ \\
\hline OME & $11(58 \%)$ \\
\hline \multicolumn{2}{|l|}{ Participants with other ear symptoms during study period, n (\%) } \\
\hline \multicolumn{2}{|l|}{ Dry perforation } \\
\hline Eustachian tube dysfunction & $4(22 \%)$ \\
\hline Granular myringitis & $1(5 \%)$ \\
\hline Participants with no OM or ear symptoms during study period, n (\%) & $1(5 \%)$ \\
\hline \multicolumn{2}{|l|}{ Participants receiving pharmaceutical treatment during study, n (\%) } \\
\hline Ciprofloxacin drops & $10(53 \%)$ \\
\hline Oral antibiotics & $9(47 \%)$ \\
\hline \multicolumn{2}{|l|}{ Participants receiving surgical treatment during study, n (\%) } \\
\hline Grommet insertion & $5(26 \%)$ \\
\hline Adenoidectomy & $7(37 \%)$ \\
\hline
\end{tabular}

Abbreviations: $A O M$ acute otitis media, $A O M w P$ acute otitis media with perforation, CSOM chronic suppurative otitis media, OM otitis media, OME otitis media with effusion 


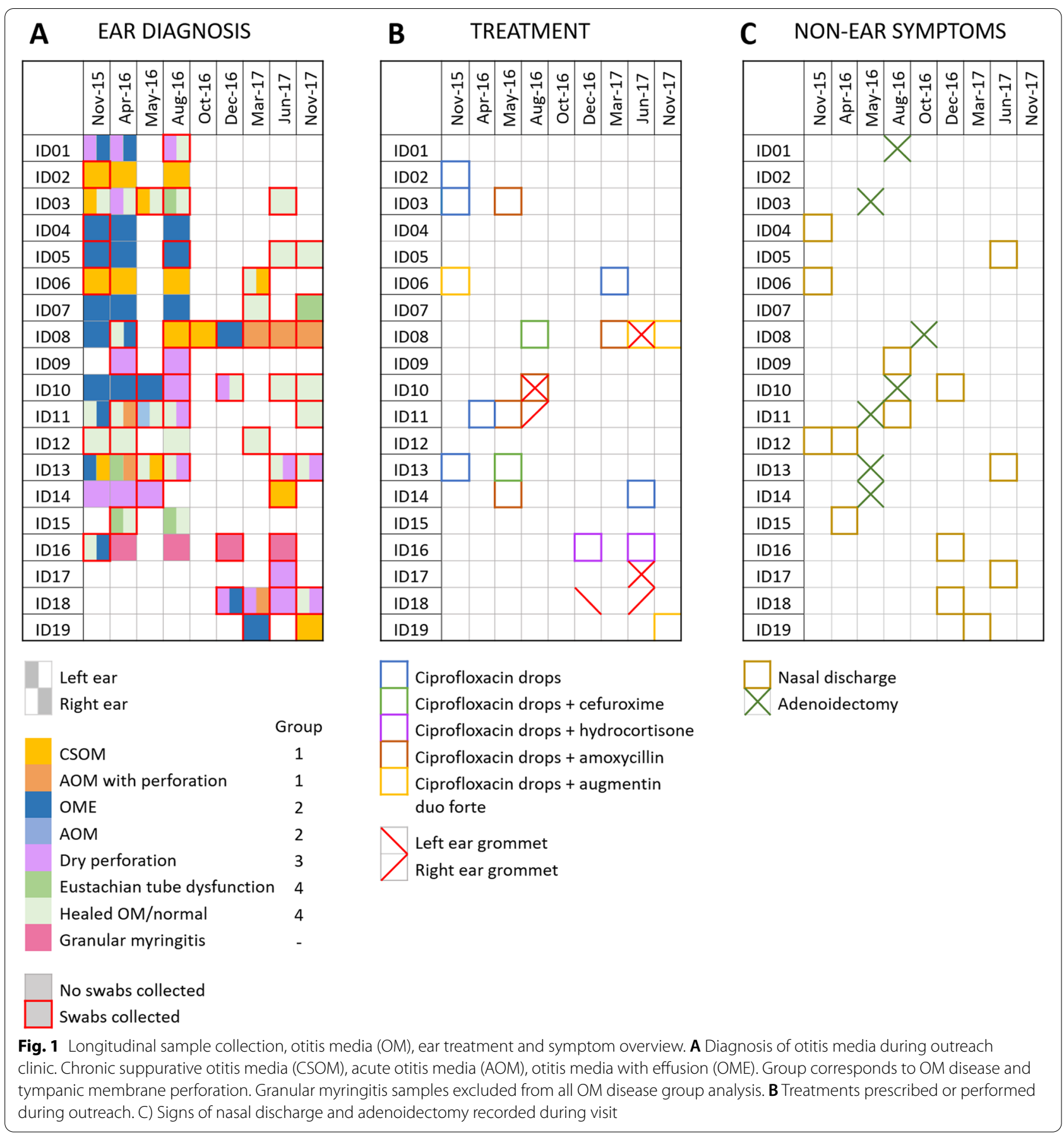

of the same flocked swabs inserted into the nasal cavity along the floor of the nose and directed posteriorly towards the nasopharynx as far as tolerated by the child. Effort was made to minimise contact with external skin to avoid contaminating skin flora. All swabs were stored without storage media (dry) and placed directly into the clinic freezer for the duration of the visit. Swabs were transported on ice in an insulated ice chest for up to 4 hours before being stored at $-80^{\circ} \mathrm{C}$ until processed. Culture-based analysis of the swabs was not performed as swabs were frozen soon after collection to preserve microbial DNA.

\section{Otitis media and hearing assessment and management}

All children who presented to the clinic underwent a medical assessment and a screening hearing assessment, 
regardless of whether they were enrolled as a participant in the study. OM was diagnosed by the visiting otolaryngologist specialist. Clinical and audiological data were correlated and if medical intervention was required then children were commenced on appropriate oral and/or topical therapy for OM. Targeted medical therapy followed the therapeutic guidelines that were current at the time of the child being seen. Escalation of therapy occurred if children had been prescribed standard medical treatment prior to specialist review by staff in the health clinic, and if there was ongoing evidence of chronic ear discharge.

For the purpose of assessing the association between ear microbiology and ear disease, ear condition was categorised into four discrete diagnosis groups. Group 1 included OM with inflammation with tympanic membrane perforation (e.g. CSOM, AOM with perforation). Group 2 included of inflammation without perforation (e.g. OME, AOM). Group 3 included no inflammation with tympanic membrane perforation (e.g. dry perforation). Group 4 included no inflammation and no perforation (e.g. normal or healed tympanic membrane).

\section{DNA extraction and $16 \mathrm{~S}$ amplicon sequencing}

Swab DNA was extracted as described previously [25] using an extraction method involving heat, mechanical, enzymatic and chemical lysis steps, followed by columnbased DNA purification (see online supplement).

The V4 hypervariable region of the bacterial 16S rRNA gene was amplified from sputum DNA using the primers: 515F (5'-GTGCCAGCMGCCGCGGTAA-3') and 806R (5'-GGACTACHVGGGTWTCTAAT') with Illumina adapter overhang sequences as previously described [26]. Amplicons were generated, cleaned, and indexed, and paired end sequencing was performed according to the Illumina MiSeq 16S Metagenomic Sequencing Library Preparation protocol. Sequencing data are deposited in the European Nucleotide Archive database (PRJEB42298).

\section{Bioinformatic processing}

Sequence output was demultiplexed using QIIME2 [27] (release 2019.4). The DADA2 plugin was used to trim, de-replicate, merge, and remove chimeric sequences, as well as identify and correct sequencing errors [28]. Representative sequences were aligned to the SILVA database (v132) at $80 \%$ using vsearch and unassigned sequences were filtered out. Remaining unique amplicon sequence variants (ASVs) were classified using the QIIME2 sklearn algorithm to the SILVA database trimmed to the V4 region of the $16 \mathrm{~S}$ rRNA gene at $97 \%$ sequence similarity. The resulting taxa (ASVs that aligned to SILVA to the genus level or higher) were then examined. Taxa that were Mitochondria, Chloroplast, Cyanobacteria, or Archaea were filtered out, as were taxa present at $<1 \%$ relative abundance in at least 1 sample. Following taxa filtering, it was noted that some of the bacterial taxa identified are potential contaminants [29]. However, these taxa are also plausible constituents of the samples and as such these taxa were denoted by an asterisk (*) where presented in our findings. Samples with fewer reads than the rarefaction plot asymptote (600 reads) were removed, which included 3 ear swabs and 3 nasopharyngeal swabs.

\section{Quantitative PCR}

Enumeration of bacteria Moraxella catarrhalis and Haemophilus influenzae, and fungi Candida albicans and Aspergillus fumigatus were performed using quantitative PCR (qPCR) as described previously [30-33] and provided in detail in the online supplement.

\section{Statistical analysis and visualisation}

Genus-level relative abundance data were square-root transformed and Bray-Curtis similarity scores calculated using the vegan $R$ package (version 2.5-7). Between group differences in Bray-Curtis scores were analysed using permutational multivariate analysis of variance (PERMANOVA). Principle co-ordinate analysis (PCoA) and non-metric multi-dimensional scaling (nMDS) plots were used to visualise Bray-Curtis distances, calculated using vegan and visualised using ggplot2 (version 3.3.3). Linear discriminant analysis (LDA) Effect Size (LEfSe) was used to assess relative abundance differences between groups, using one-against-all multi-class analysis, and cut-offs of LDA $\geq 3$ and $p<0.05$. Kruskal-Wallis test with Dunn's post-hoc analysis used to validate LEfSe findings, as well as compare species level qPCR results and age between groups.

\section{Results \\ Cohort overview and otitis media prevalence}

Fifty-one sets of swab samples (left ear canal, right ear canal, and nasopharynx) were collected from 19 children presenting to Tullawon Health Clinic in remote Western South Australia over a two-year period. Multiple sets of samples were collected longitudinally from 19 children with the age range at the time of first swab collection was between 3 months and 8.7 years (median $[\mathrm{IQR}]=3.2$ [1.7-4.9], Table 1).

The middle ear and hearing health status for each child was assessed at every visit during which samples were collected (Fig. 1A). From a total of 102 ear diagnoses (left and right ear during 51 examinations), signs of $\mathrm{OM}$ with symptomatic tympanic membrane perforation were identified in 22 cases (15 CSOM and 7 AOM with perforation or AOM with grommet), asymptomatic tympanic 
membrane perforations were diagnosed in 20 (12 dry perforation, 8 dry grommet), signs of OM without perforation in 16 ( $1 \mathrm{AOM}, 15 \mathrm{OME})$, and no signs of current disease or perforation in 36 (15 healed OM, 21 normal ear). Granular myringitis was diagnosed in 4 cases ( 2 visits of bilateral disease in 1 participant) and eustachian tube dysfunction in 4 cases from 3 participants. Rates of unilateral and bilateral OM presentation were similar (42 and $47 \%$, respectively).

Treatment was primarily ciprofloxacin drops alone $(n=8)$, or ciprofloxacin drops with oral antibiotics $(n=7)$, and surgical insertion of grommets $(n=9)$. An overview of all interventions is provided in Fig. 1B and non-ear symptoms in Fig. 1C.

\section{Disease heterogeneity within ear samples longitudinally}

Within-participant changes in diagnosis are presented as a network plot (Supplementary Fig. 1). In the majority of cases, diagnoses were consistent between visits, with 74 of $114(65 \%)$ of consecutive examinations resulting in the same diagnosis, compared to $40 / 114$ instances where diagnosis changed (Supplementary Fig. 1). Of the instances where diagnosis changed, OME recovered to normal four times, dry perforation recovered to healed four times, while there were three instances where OME progressed to CSOM, and three instances where dry perforation progressed to CSOM. Overall, however, no one transition disease phenotype was predominant.

\section{Ear microbiota characteristics}

Analysis of the ear swab microbiota identified 134 bacterial taxa at $\geq 1 \%$ relative abundance in at least one sample. Of these, $20 \%$ were Actinobacteria, $14 \%$ Bacteroidetes, 35\% Firmicutes, 25\% Proteobacteria, and 6\% other bacterial phyla. A median of 40 taxa were identified within any individual sample (IQR: 30-53), representing approximately $30 \%$ of all detected taxa. While this finding is consistent with considerable variation in microbiota composition between samples, several taxa were commonly detected. Corynebacterium was identified most frequently $(70 \%$ of ear swabs at a mean relative abundance of $7.8 \%$ ), while Alloiococcus, was found at the highest mean relative abundance (present in $58 \%$ of ear swabs at a mean relative abundance of 27\%). Staphylococcus, Haemophilus, Turicella, Streptococcus, and Pseudomonas were all detected in at least $50 \%$ of samples and had mean relative abundances of $11,6.5,9.0,1.3$, and $3.2 \%$, respectively (Fig. 2A). Taxa bar plots of all ear and nose samples are presented in Supplementary Fig. 2. Analysis of fungi Candida albicans and Aspergillus fumigatus was also performed. However, only $A$. fumigatus was detected and only in one ear swab.

\section{Variation in microbiota composition within and between individuals}

Similarity in ear microbiota were calculated for all samples using a Bray-Curtis similarity matrix (BC distance), where a distance score between 0 and 1 indicates similarity (most similar $=1$, least $\operatorname{similar}=0$ ). Based on the first sample collected from each participant, the microbiota of a subject's ear was more similar to their other ear than to swabs from other participants (median BC distance score within participant $=0.22$ (IQR: $0.52-0.07)$ vs between participant $=0.04(\mathrm{IQR}$ :
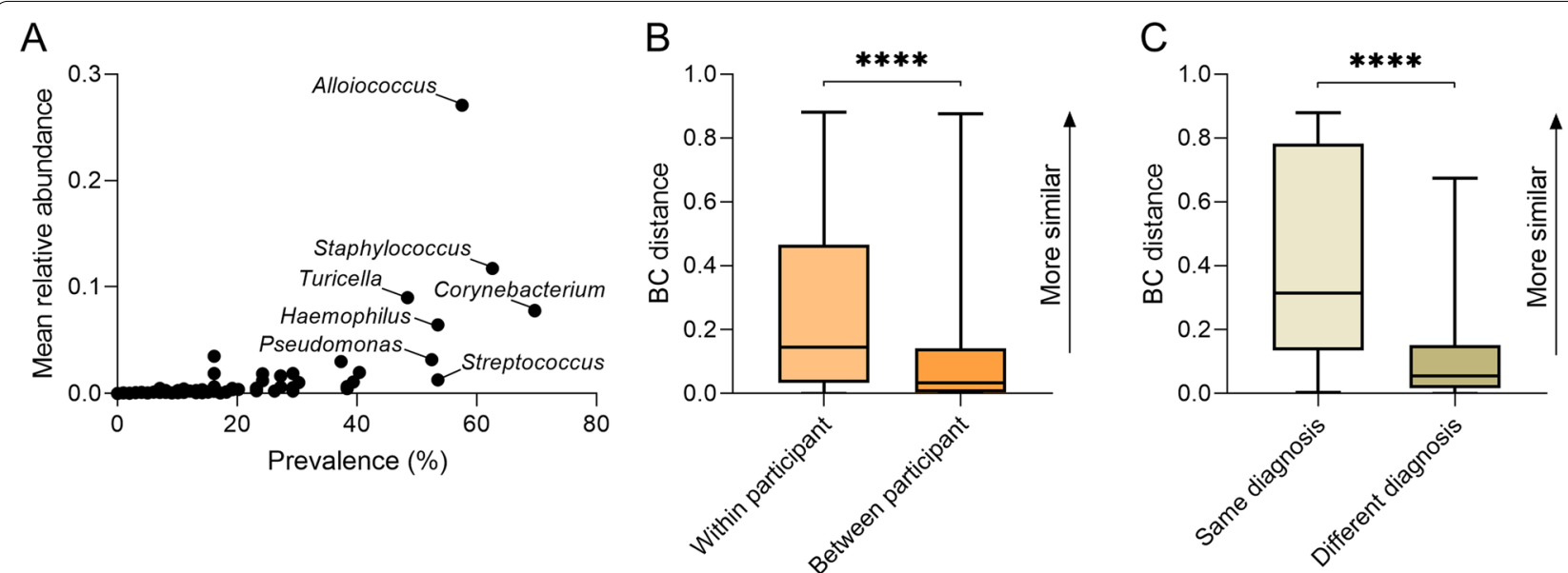

Fig. 2 A Mean relative abundance and detection prevalence (\%) of taxa in ear swabs. Labelled taxa indicate presence in at least $50 \%$ of ear swab samples. B Bray-Curtis similarity (BC distance) of ear microbiota comparing swabs collected within individuals to those collected between individuals, C) Longitudinal ear microbiota similarity for ears that had the same diagnosis between visits vs ears that had different diagnoses between visits 


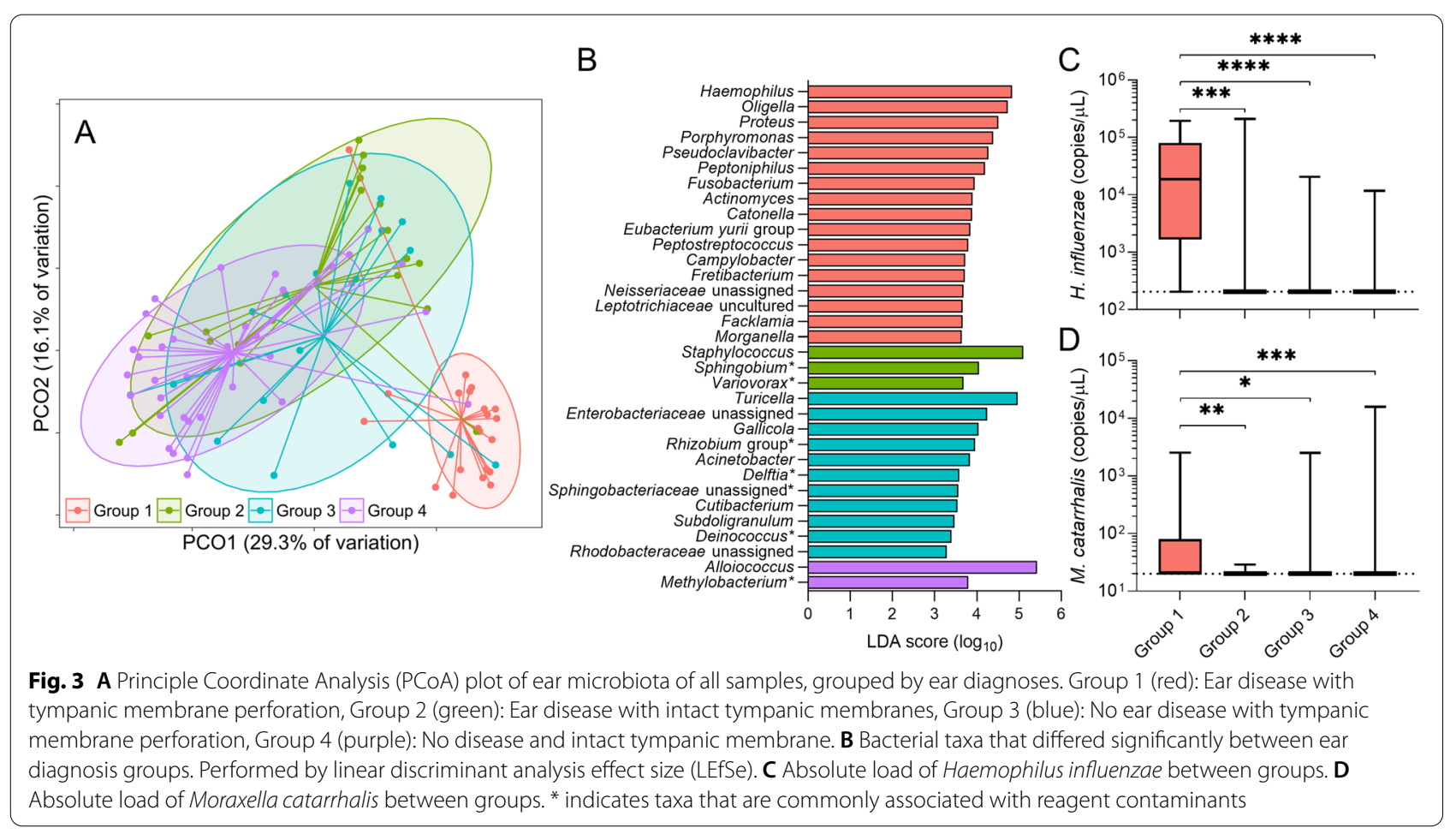

$0.14-0) ; p=0.0006)$. This finding remained true when all time points were assessed (median BC distance score within participant $=0.14$ (IQR: $0.46-0.03)$ vs between participant $=0.03$ (IQR: 0.14-0); $p<0.0001$, Fig. 2B). When stratified according to clinical diagnosis (e.g. CSOM, AOMwP, AOM, OME, dry perf, eustachian tube dysfunction or normal), microbiota similarity was greatest between the left and the right ear for an individual when the diagnosis was the same. However, even when the diagnoses were different, microbiota similarity was still significantly higher within an individual compared to between individuals, and was non-significantly higher than between individuals, even where individuals had the same diagnosis (Supplementary Fig. 3A).

Longitudinal analysis of ear swab microbiota similarity was then performed. Where ear swabs were compared between two timepoints, they were more similar if the diagnosis remained the same, as compared to instances where diagnosis changed (Fig. 2C). This finding was unaffected by the time interval between examinations, with a weak correlation between time between samples and BC distance (Supplementary Fig. 3B). Taken together, these findings indicate that both participant heterogeneity and disease diagnosis contribute to observed ear microbiota.

\section{Ear microbiota, OM symptoms, and tympanic membrane integrity}

Ear microbiota characteristics were assessed relative to four discrete diagnosis groups: 1) inflammation with tympanic membrane perforation $(n=21)$; 2$)$ inflammation without perforation $(n=20)$; 3) no inflammation with tympanic membrane perforation $(n=18) ; 4)$ no inflammation and no perforation $(n=36)$. Microbiota composition differed significantly between Groups (PERMANOVA: $r^{2}=21.2 \%$, P $($ perm $\left.)=0.0001\right)$. Of note, Group 1 (inflammation with tympanic membrane perforation) showed the greatest difference to the other Groups (Supplementary Table 1) and was found to cluster separately (Fig. 3A). It should be noted that the age and sex profiles of the four groups differed (Supplementary Fig. 3C and 3D). Those in diagnosis Group 1 and Group 2 were significantly younger than Group 3 and Group 4, while females had a higher prevalence of having perforated ear disease (Group 1) over the study period.

The microbiota composition of the ear swabs is shown in Supplementary Fig. 4, grouped according to the four diagnosis categories. LEfSe analysis identified 33 taxa as contributing significantly to inter-group differences. For example, the genus Haemophilus was particularly prevalent in Group 1, while Staphylococcus, Turicella, and Alloiococcus were predominant in Groups 2, 3 and 4, respectively (Fig. 3B). These findings were confirmed by Kruskal-Wallis test with Dunn's post-hoc analysis 

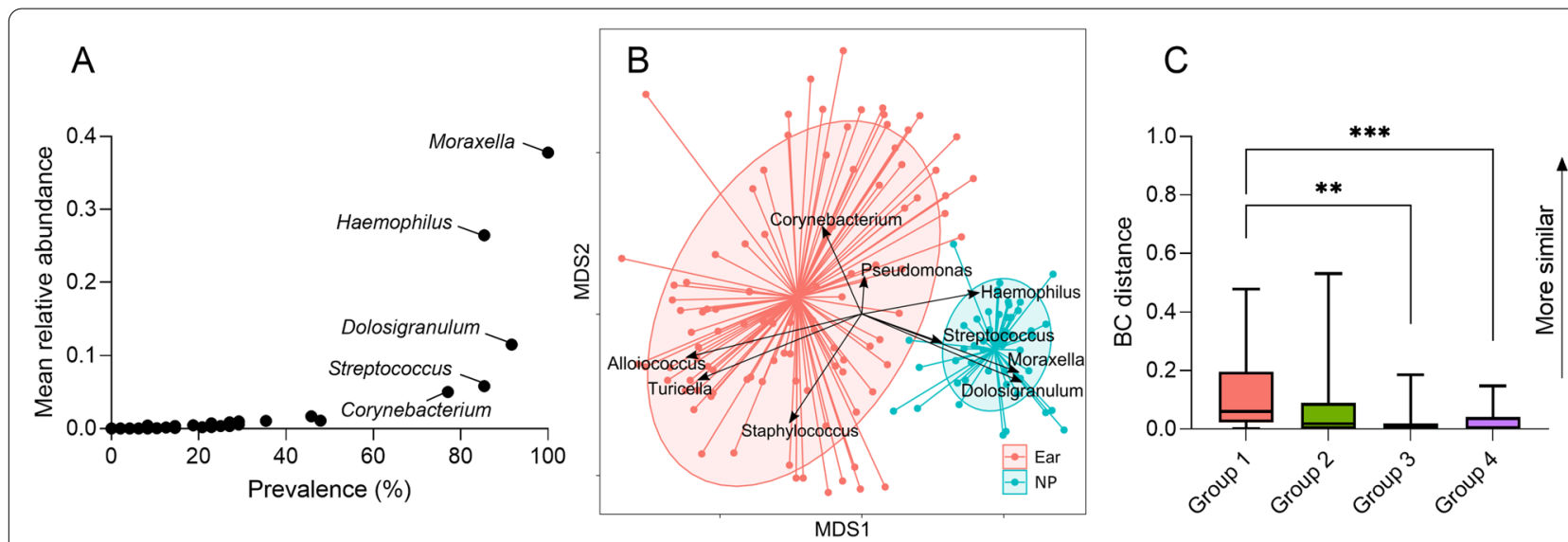

Fig. 4 A Mean relative abundance and detection prevalence (\%) of bacteria in nasopharyngeal (NP) swabs. B Non-metric multi-dimensional scaling (NMDS) biplot of ear and nasopharyngeal microbiota. C Bray-Curtis similarity (BC distance) between nasopharyngeal microbiota and ear microbiota, separated by ear disease groups. Group 1: Ear disease with tympanic membrane perforation, Group 2: Ear disease with intact tympanic membranes, Group 3: No ear disease with tympanic membrane perforation, Group 4: No disease and intact tympanic membrane

(Supplementary Fig. 5). Exploratory species level analysis for Staphylococcus spp. identified that the relative abundance of Staphylococcus aureus was significantly higher in Group 2 and Group 4 compared to Group 1 (Supplementary Fig. 6). Of note, Oligella, a Gram-negative, aerobic Proteobacteria, which is not commonly associated with human respiratory or ear infections [34], was a substantial contributor to Group 1 microbiota characteristics. Targeted qPCR-based assessment of $H$. influenzae and $M$. catarrhalis carriage confirmed absolute abundance of these species to be higher in Group 1 compared to other diagnostics groups (Fig. 3C-D).

\section{Nasopharyngeal microbiota characteristics}

Microbiota profiles generated from nasopharyngeal swabs were sparser and more consistent between subjects than ear sample profiles. Eighty-eight taxa were detected across all nasopharyngeal swabs, of which 53 were detected at $\geq 1 \%$ relative abundance in at least one swab. Compared to ear swabs, more taxa were common across samples, with a median of 37 taxa per swab (IQR: 29-48). These taxa represented Firmicutes (31\%), Proteobacteria (31\%), Actinobacteria (17\%), Bacteroidetes (16\%), and other phyla (5\%). Moraxella was detected in all nasopharyngeal samples and displayed the highest mean relative abundance at 38\% (Fig. 4A, Supplementary Fig. 2). Haemophilus, Dolosigranulum, Streptococcus, and Corynebacterium were all detected in at least $50 \%$ of samples with mean relative abundances of $26,12,5.8$, and $5.0 \%$, respectively (Fig. 4A, Supplementary Fig. 2).

Nasopharyngeal microbiota differed significantly in composition to ear microbiota (PERMANOVA: $r^{2}=24.5 \%, \mathrm{P}($ perm $\left.)=0.0001\right)$. This difference was driven primarily by higher relative abundance of Alloiococcus, Turicella, Corynebacterium, and Pseudomonas in ear swabs and higher relative abundance of Moraxella, Dolosigranulum, Streptococcus, and Haemophilus in the nasopharyngeal swabs (Fig. 4B).

Nasopharyngeal microbiota showed little similarity to ear microbiota for individual participants (median BC distance $=0.015$ (IQR: 0.06-0). However, this relationship differed between diagnosis groups, with nasopharyngeal microbiota more similar to the ear microbiota in Group 1 (inflammation with tympanic membrane perforation) compared to Group 3 (no inflammation with tympanic membrane perforation) or Group 4 (no inflammation, no perforation) (Fig. 4C). The abundance of Haemophilus in Group 1 ear swabs was the likely principal contributor to this, with reciprocal abundance typical in nasopharyngeal swabs. To explore this further, Haemophilus relative abundance in ear and nose swabs were correlated to the $\mathrm{BC}$ distance between these swabs for Group 1 samples. Relative abundance of Haemophilus in the 21 ear swabs with inflammation with tympanic membrane perforation was positively correlated with $\mathrm{BC}$ distance between ear and nasopharyngeal sample $\left(r_{\mathrm{s}}=0.71, p=0.0003\right)$, indicating that high Haemophilus in the ears was associated with a greater similarity between ear and nasopharyngeal microbiota.

\section{Relationships between disease, treatment, and ear microbiota over time}

Microbiota characteristics from a single individual (ID08), collected on seven successive occasions during a 19-month period (aged 7 months to 2 years 2 months), were related to disease characteristics and treatment. 
During this period, an initial presentation of unilateral OME progressed to bilateral CSOM, reverted to OME, and then developed into AOM with perforation (Fig. 1). In response, a step-up therapy approach was employed. No treatment was prescribed for initial OME, ciprofloxacin drops plus oral cefuroxime was prescribed at first presentation with CSOM (the diagnosis of which was based upon reported duration of discharge) (Fig. 1), and ciprofloxacin drops with oral amoxicillin once AOM with perforation was diagnosed. As symptoms continued, grommets were inserted, and oral amoxicillin continued. At the last study visit, oral co-amoxiclav (amoxicillin/clavulanic acid) and topical ciprofloxacin were prescribed.

Changes in clinical signs and treatment were reflected in ear canal microbiology (Fig. 5). Initial unilateral OME was associated with a high prevalence of Staphylococcus (78\%). Throughout interventions for bilateral CSOM the predominant taxa changed from bilateral Haemophilus (68-73\% relative abundance) to Corynebacterium
(47-80\% relative abundance) once the tympanic membranes had healed and OME developed, to Pseudomonas (41-52\%) when AOM with perforation was observed. At the time of procedure, when grommets were inserted, the relative abundance of Pseudomonas had declined (0-5\%), while Haemophilus relative abundance increased reciprocally $(47-54 \%)$. Finally, at the consult 5 months after grommet insertion, Oligella was prevalent in both ear swabs (40-60\% relative abundance).

\section{Discussion}

We report an exploration of the incidence and clinical and microbiological characteristics of $\mathrm{OM}$ in children presenting at a South Australian outreach clinic over a two-year period. The aim of this study was to provide a basis to re-examine OM prevention and treatment strategies for this community and remote Aboriginal communities more widely.

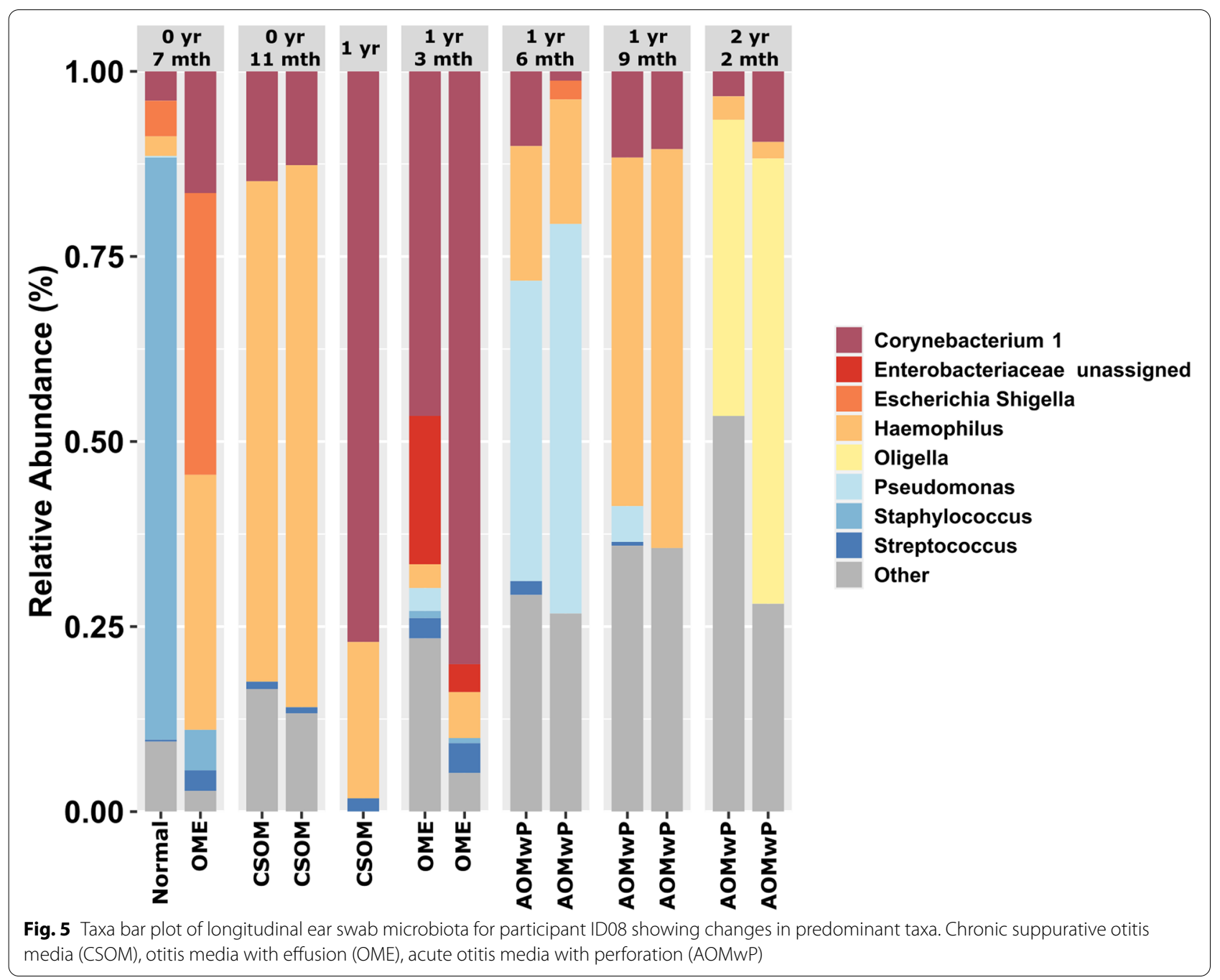


Study participants exhibited ear disease that aligned with other studies of Aboriginal children living in remote communities, over a third of children in this study exhibiting CSOM over the study period [5]. OME was also seen in over half of these children. The types of ear disease exhibited by study participants varied with age and comparable rates of unilateral and bilateral OM presentations are also consistent with national data [35].

Recognition of the potential for a wider spectrum of bacteria to contribute to OM pathology, as well as for the wider microbial communities to positively and negatively influence the behaviour of pathogens [17, 24], has resulted in a growing application of microbiota-wide analysis to OM samples [18]. Most commonly, these take the form of 16S rRNA gene amplicon sequencing, which, when applied in an appropriate manner [24, 36, 37], can provide detailed compositional data relating to all bacteria present. The composition of the ear microbiota reported here are broadly consistent with previous studies. We identified Alloiococcus, Staphylococcus, and Turicella as prevalent, in keeping with studies of middle ear fluid of children living in metropolitan regions with recurrent AOM [24], as well as Aboriginal children in Central Australia [36]. Whether Alloiococcus and Turicella are otopathogenic or part of the normal aural "flora" remains contentious [24]. We found that Alloiococcus was more prevalent in the ear canals of children without current OM, while Turicella was more prevalent in those with either no OM or a dry perforation. Staphylococcus aureus, on the other hand, was significantly over-represented in ear canals of those with OME and was significantly higher than those with either CSOM or AOM with perforation. Further research is required to determine whether S. aureus is indicative or contributory to OME.

In keeping with previous studies in Aboriginal children in remote Australia [17], and globally [38, 39], we found $H$. influenzae, and to a lesser extent, $M$. catarrhalis, to be associated with chronic and perforated OM. We also found Oligella to be significantly more abundant in those with ear disease with perforation, detected in $16 \%$ of all ear swabs and with a relative abundance up to $60 \%$. Oligella consists of 2 Gram-negative, aerobic species associated with urosepsis, Oligella urethralis and Oligella ureolytica. However, Oligella is rarely associated with OM [34] although it has been reported at high abundance in middle ear swabs from indigenous Filipinos with chronic otitis media [40]. The potential for Oligella to be an important contributor to OM in Aboriginal children clearly requires further investigation. Indeed, it is notable that ciprofloxacin-resistant clinical isolates of Oligella have been reported in a number of clinical contexts [41-43].
Based on longitudinal analysis, we reported that ear microbiota composition varies greatly within individuals. In participant ID08, for example, the predominant organism varied between Corynebacterium, Pseudomonas, Haemophilus, and Oligella, within a 11-month window. These shifts in which organisms were predominant also co-occurred with changes in diagnosis and treatment. Whether changes in microbiology preceded changes in disease, or vice versa, remains unclear. However, these findings do suggest that high rates of OM in Aboriginal communities cannot be explained by a single un-recognised otopathogen.

It has been suggested that acute upper respiratory tract infections (URTI) may represent an important contributory factor in OM development, directly through infection of the middle ear, by promoting the build-up of fluid in the middle ear, or by promoting the proliferation of opportunistic pathogens within the upper respiratory tract $[44,45]$. Rates of URTI are significantly higher in Aboriginal children [46] and might therefore contribute to high OM incidence. There was little similarity between nasopharyngeal swab and ear swab microbiota composition, in keeping with previous studies [24, 36]. However, we did find that the ear canal microbiota was more similar to the nasopharyngeal microbiota in those children in whom the tympanic membrane had been ruptured (Group 1). This increase in similarity was driven largely by a high relative abundance of the otopathogen, $H$. influenzae, which trended strongly towards increased relative and absolute abundance in nasopharyngeal samples of those with respiratory symptoms. Disruption of commensal microbiology and local immune homeostasis by acute respiratory viral infection can increase the relative abundance of opportunist bacterial pathogens and the likelihood of bacterial infection in the upper respiratory tract and adjoining regions such as the Eustachian tube and middle ear cleft [44]. Therefore, whether high rates of URTI in Aboriginal children, including those caused by respiratory viral infection, are causally associated with high rates of bacterial infection in the middle ear and lower respiratory tract, should be considered.

It is notable that the bacterial species detected in our analysis, including those present in the ear canal in children with perforated tympanic membranes, sit within the spectrum of activity of standard antibiotic therapies for OM [15]. Where OM is recurrent, the surgical insertion of a grommet into the tympanic membrane can be used to prevent the build-up of fluid in the middle ear (a measure that was performed in just over a quarter of the study participants during the period of investigation). Therefore, rather than standard therapies being inappropriate for this population, high OM incidence, duration, and severity are likely to reflect a combination of high 
exposure to environmental risk factors, poorly understood treatment adherence, and limited access to specialist otolaryngology services [47-49].

Our study had limitations that should be considered. Microbiological analysis was based on molecular analysis from swab samples collected from the ear canal, with culture-based analysis not performed. While OM diagnosis and antibiotic prescription are based largely on otoscopy and assessment of signs and symptoms, ear canal swabs can be used as a basis of diagnostic microbiology, particularly where treatment response has been poor, there is exudate in the ear canal, or where there is otitis externa (inflammation of the passage of the outer ear). Ear canal swabs clearly do not sample material from the middle ear in those with intact tympanic membranes. Sampling middle ear fluid through myringotomy would require sedation or a general anaesthetic, which is not standard care unless examination requires surgical intervention. Particularly in remote communities such as Yalata, the required resources for such procedures is not available for therapeutic or research practices. Despite the sample site being ear canals, studies comparing the ear canal microbiota to the middle ear fluid of children with AOM identified surprisingly similar composition between the two sites, although Haemophilus was under-represented in the ear canal [24]. Being a safe, well-tolerated, and readily employed approach, ear canal samples, when combined with a history of duration of discharge, do have potential applicability as a representative sample of the middle ear microbiota in the context of perforation, or even as a prognostic indicator. In addition to bacterial and fungal pathogens, respiratory viruses can contribute substantially to $\mathrm{OM}$ development and progression [16], however viruses were not investigated in this study. Assessment of antibiotic susceptibility was not part of our study. However, the ability to detect the presence of antimicrobial resistance with a specific child or within a community would allow for tailoring of antimicrobial choice, and such data would inform our understanding of treatment efficacy. Finally, all analysis was performed without the adjustment for confounding factors such as age or sex owing to the exploratory nature of this study. Further, larger studies relating the ear microbiome to $\mathrm{OM}$ are required to assess the contribution of age and sex to these associations.

\section{Conclusions}

$\mathrm{OM}$ has a considerable and long-lasting impact on the health, education and the future prospects of Australian Aboriginal children, particularly for those living in remote communities. Addressing this disease burden is complex and challenging, requiring consideration of a wide range of potential contributors, including genetic, environmental, socioeconomic and demographic factors, access to healthcare, and microbial ecology. Our use of a microbiome-focused molecular strategy to describe the heterogeneity and variance of ear canal microbiota and its associations with disease and treatment, highlight the potential utility of such an approach as part of the ongoing wider effort to better understand, prevent, and manage $\mathrm{OM}$ in Aboriginal children.

\begin{abstract}
Abbreviations
AHW: Aboriginal Health Workers; AOM: Acute otitis media; AOMwP: Acute otitis media with perforation; ASV: Amplicon sequence variant; BC Distance: Bray-Curtis similarity distance; OM: Otitis media; CSOM: Chronic suppurative otitis media; LEfSe: Linear discriminant analysis effect size; nMDS: Non-metric multi-dimensional scaling; OME: Otitis media with effusion; PERMANOVA: Permutational multivariate analysis of variance; PCOA: Principle co-ordinate analysis; qPCR: Quantitative PCR; rRNA: Ribosomal ribonucleic acid; URTI: Acute upper respiratory tract infections.
\end{abstract}

\section{Supplementary Information}

The online version contains supplementary material available at https://doi. org/10.1186/s12866-022-02436-x.

Additional file 1.

\section{Acknowledgements}

We thank all the children and their families who participated in this study. We also thank Gary Goldsmith, the Deadly Sounds Coordinator with the Aboriginal Health Council of South Australia during the study, who helped with community engagement and recruitment, and all the nursing staff and Aboriginal Health Workers at the Tullawon Health Clinic.

\section{Authors' contributions}

PM and GBR conceived the study. PM and CW designed the study. PM collected the samples, recruited participants and participant data. AR, JMC, and FA extracted DNA from the samples and performed 16S sequencing. WXK performed the qPCR. SLT analysed the sequencing and qPCR output and undertook the statistical analysis. SLT, GBR, and PM interpreted the data. LEP and JMC provided qPCR methodology. PM, SW, EO, and LEP provided clinical insight and interpretation. SLT, GBR, and PM wrote the manuscript. All authors read and approved the final manuscript.

\section{Funding}

This study was funded by the Centre of Research Excellence in Ear and Hearing Health of Aboriginal and Torres Strait Islander Children (CRE ICHEAR, NHMRC APP1078557), The South Australian Health and Medical Research Institute internal funding, and The Flinders Medical ENT Unit (SALHN 40-803-8021515). GBR was supported by a Matthew Flinders Research Fellow and a NHMRC Senior Research Fellowship (GNT1155179).

\section{Availability of data and materials}

The dataset generated and analysed during the current study is available in the European Nucleotide Archive database (PRJEB42298; available at www.ebi. ac.uk/ena/browser/view/PRJEB42298) and additional data is available from the corresponding author on request.

\section{Declarations}

Ethics approval and consent to participate

Ethics approval for this study was obtained from the Southern Adelaide Clinical Human Research Ethics Committee (249.15) and the Aboriginal Health Research Ethics Committee (04-15-615). All parents and guardians of participants provided written informed consent. 


\section{Consent for publication}

Not applicable.

\section{Competing interests}

The authors declare that they have no competing interests.

\section{Author details}

'Microbiome and Host Health, South Australian Health and Medical Research Institute, Adelaide, South Australia, Australia. ${ }^{2}$ College of Medicine and Public Health, Flinders University, Adelaide, South Australia, Australia. ${ }^{3}$ Department of Otolaryngology, Head \& Neck Surgery, Flinders Medical Centre, Adelaide, South Australia, Australia. ${ }^{4}$ South Australian Health and Medical Research Institute, Adelaide, South Australia, Australia.

Received: 30 July 2021 Accepted: 29 December 2021

Published online: 13 January 2022

\section{References}

1. Kong K, Coates HL. Natural history, definitions, risk factors and burden of otitis media. Med J Aust. 2009;191(S9):S39-43.

2. Coticchia JM, Chen M, Sachdeva L, Mutchnick S. New paradigms in the pathogenesis of otitis media in children. Front Pediatr. 2013;1:52.

3. Daly KA, Hoffman HJ, Kvaerner KJ, Kvestad E, Casselbrant ML, Homoe P, et al. Epidemiology, natural history, and risk factors: panel report from the ninth international research conference on otitis media. Int J Pediatr Otorhinolaryngol. 2010;74(3):231-40.

4. Bhutta MF. Evolution and otitis media: a review, and a model to explain high prevalence in indigenous populations. Hum Biol. 2015;87(2):92-108.

5. Morris PS, Leach AJ, Silberberg P, Mellon G, Wilson C, Hamilton E, et al. Otitis media in young Aboriginal children from remote communities in northern and Central Australia: a cross-sectional survey. BMC Pediatr. 2005;5:27.

6. World Health Organization. Chronic suppurative otitis media: burden of illness and management options. 2004.

7. Russell DJ, Zhao Y, Guthridge S, Ramjan M, Jones MP, Humphreys JS, et al. Patterns of resident health workforce turnover and retention in remote communities of the Northern Territory of Australia, 2013-2015. Hum Resour Health. 2017;15(1):52.

8. Australian Institute of Health and Welfare. Indigenous hearing health. Canberra: AlHW: 2020

9. Su JY, He VY, Guthridge S, Howard D, Leach A, Silburn S. The impact of hearing impairment on Aboriginal children's school attendance in remote Northern Territory: a data linkage study. Aust N Z J Public Health. 2019:43(6):544-50.

10. Su JY, Guthridge S, He VY, Howard D, Leach AJ. The impact of hearing impairment on early academic achievement in Aboriginal children living in remote Australia: a data linkage study. BMC Public Health. 2020;20(1):1521.

11. Su JY, Guthridge S, He VY, Howard D, Leach AJ. Impact of hearing impairment on early childhood development in Australian Aboriginal children: a data linkage study. J Paediatr Child Health. 2020;56(10):1597-606.

12. Anderson ML, Chang BH, Kini N. Alcohol and drug use among deaf and hard-of-hearing individuals: a secondary analysis of NHANES 2013-2014. Subst Abus. 2018:39(3):390-7.

13. Clearinghouse TCtG. Ear disease in Aboriginal and Torres Strait islander children. Canberra: AlHW; 2014.

14. Williams CJ, Jacobs AM. The impact of otitis media on cognitive and educational outcomes. Med J Aust. 2009;191(S9):S69-72.

15. Leach AJ, Morris PS, Coates HL, Nelson S, O'Leary SJ, Richmond PC, et al. Otitis media guidelines for Australian Aboriginal and Torres Strait islander children: summary of recommendations. Med J Aust. 2021;214(5):228-33.

16. Massa HM, Cripps AW, Lehmann D. Otitis media: viruses, bacteria, biofilms and vaccines. Med J Aust. 2009;191(S9):S44-9.

17. Smith-Vaughan HC, Binks MJ, Marsh RL, Kaestli M, Ward L, Hare KM, et al. Dominance of Haemophilus influenzae in ear discharge from indigenous Australian children with acute otitis media with tympanic membrane perforation. BMC Ear Nose Throat Disord. 2013;13(1):12.

18. Coleman A, Wood A, Bialasiewicz S, Ware RS, Marsh RL, Cervin A. The unsolved problem of otitis media in indigenous populations: a systematic review of upper respiratory and middle ear microbiology in indigenous children with otitis media. Microbiome. 2018;6(1):199.

19. Leach AJ, Boswell JB, Asche V, Nienhuys TG, Mathews JD. Bacterial colonization of the nasopharynx predicts very early onset and persistence of otitis media in Australian Aboriginal infants. Pediatr Infect Dis J. 1994;13(11):983-9.

20. Sun W, Jacoby P, Riley TV, Bowman J, Leach AJ, Coates H, et al. Association between early bacterial carriage and otitis media in Aboriginal and non-Aboriginal children in a semi-arid area of Western Australia: a cohort study. BMC Infect Dis. 2012;12(1):366.

21. Leach AJ, Morris PS. Antibiotics for the prevention of acute and chronic suppurative otitis media in children. Cochrane Database Syst Rev. 2006:(4):CD004401.

22. Leach AJ, Wigger C, Beissbarth J, Woltring D, Andrews R, Chatfield MD, et al. General health, otitis media, nasopharyngeal carriage and middle ear microbiology in Northern Territory Aboriginal children vaccinated during consecutive periods of 10-valent or 13-valent pneumococcal conjugate vaccines. Int J Pediatr Otorhinolaryngol. 2016;86:224-32.

23. Nicholls TR, Leach AJ, Morris PS. The short-term impact of each primary dose of pneumococcal conjugate vaccine on nasopharyngeal carriage: systematic review and meta-analyses of randomised controlled trials. Vaccine. 2016;34(6):703-13.

24. Lappan R, Imbrogno K, Sikazwe C, Anderson D, Mok D, Coates H, et al. A microbiome case-control study of recurrent acute otitis media identified potentially protective bacterial genera. BMC Microbiol. 2018;18(1):13.

25. Choo JM, Abell GCJ, Thomson R, Morgan L, Waterer G, Gordon DL, et al. Impact of long-term erythromycin therapy on the Oropharyngeal microbiome and resistance gene reservoir in non-cystic fibrosis bronchiectasis. Msphere. 2018;3(2):e00103-18.

26. Douglas CA, Ivey KL, Papanicolas LE, Best KP, Muhlhausler BS, Rogers GB. DNA extraction approaches substantially influence the assessment of the human breast milk microbiome. Sci Rep. 2020;10(1):123.

27. Bolyen E, Rideout JR, Dillon MR, Bokulich NA, Abnet CC, Al-Ghalith GA, et al. Reproducible, interactive, scalable and extensible microbiome data science using QIIME 2. Nat Biotechnol. 2019;37(8):852-7.

28. Callahan BJ, McMurdie PJ, Rosen MJ, Han AW, Johnson AJ, Holmes SP. DADA2: high-resolution sample inference from Illumina amplicon data. Nat Methods. 2016;13(7):581-3.

29. Salter SJ, Cox MJ, Turek EM, Calus ST, Cookson WO, Moffatt MF, et al. Reagent and laboratory contamination can critically impact sequence-based microbiome analyses. BMC Biol. 2014;12(1):1-12.

30. Greiner O, Day PJR, Altwegg M, Nadal D. Quantitative detection of Moraxella catarrhalis in nasopharyngeal secretions by real-time PCR. J Clin Microbiol. 2003;41(4):1386-90.

31. Innings A, Ullberg M, Johansson A, Rubin CJ, Noreus $\mathrm{N}$, Isaksson M, et al. Multiplex real-time PCR targeting the RNase P RNA gene for detection and identification of Candida species in blood. J Clin Microbiol. 2007:45(3):874-80.

32. Rantakokko-Jalava K, Laaksonen S, Issakainen J, Vauras J, Nikoskelainen J, Viljanen MK, et al. Semiquantitative detection by real-time PCR of Aspergillus fumigatus in bronchoalveolar lavage fluids and tissue biopsy specimens from patients with invasive aspergillosis. J Clin Microbiol. 2003:41(9):4304-11.

33. Reddington K, Schwenk S, Tuite N, Platt G, Davar D, Coughlan H, et al. Comparison of established diagnostic methodologies and a novel bacterial smpB real-time PCR assay for specific detection of Haemophilus influenzae isolates associated with respiratory tract infections. J Clin Microbiol. 2015;53(9):2854-60.

34. Baqi M, Mazzulli T. Oligella infections: case report and review of the literature. Can J Infect Dis. 1996;7(6):377-9.

35. Brennan-Jones CG, Hakeem HH, Costa CD, Weng W, Whitehouse AJO, Jamieson SE, et al. Cross-sectional prevalence and risk factors for otitis media and hearing loss in Australian children aged 5 to 7 years: a prospective cohort study. Aust J Otolaryngol. 2020;2020(3):8.

36. Jervis-Bardy J, Leong LE, Marri S, Smith RJ, Choo JM, Smith-Vaughan HC, et al. Deriving accurate microbiota profiles from human samples with low bacterial content through post-sequencing processing of Illumina MiSeq data. Microbiome. 2015:3:19.

37. Jervis-Bardy J, Leong LEX, Papanicolas LE, Ivey KL, Chawla S, Woods CM, et al. Examining the evidence for an adult healthy middle ear microbiome. Msphere. 2019;4(5):e00456-19. 
38. Ngo CC, Massa HM, Thornton RB, Cripps AW. Predominant Bacteria detected from the middle ear fluid of children experiencing otitis media: a systematic review. PLoS One. 2016;1 1(3):e0150949.

39. Intakorn P, Sonsuwan N, Noknu S, Moungthong G, Pircon JY, Liu Y, et al. Haemophilus influenzae type $b$ as an important cause of culture-positive acute otitis media in young children in Thailand: a tympanocentesisbased, multi-center, cross-sectional study. BMC Pediatr. 2014;14:157.

40. Santos-Cortez RL, Hutchinson DS, Ajami NJ, Reyes-Quintos MR, Tantoco ML, Labra PJ, et al. Middle ear microbiome differences in indigenous Filipinos with chronic otitis media due to a duplication in the A2ML1 gene. Infect Dis Poverty. 2016;5(1):97.

41. Riley UB, Bignardi G, Goldberg L, Johnson AP, Holmes B. Quinolone resistance in Oligella urethralis-associated chronic ambulatory peritoneal dialysis peritonitis. J Infect. 1996;32(2):155-6.

42. Pugliese A, Pacris B, Schoch PE, Cunha BA. Oligella urethralis urosepsis. Clin Infecti Dis. 1993;17(6):1069-70.

43. Lechner A, Bruckner DA. Oligella ureolytica in blood culture: contaminant or infection? Eur J Clin Microbiol Infecti Dis. 2001;20(2):142-3.

44. Hanada S, Pirzadeh M, Carver KY, Deng JC. Respiratory viral infectioninduced microbiome alterations and secondary bacterial pneumonia. Front Immunol. 2018;9:2640.

45. Ederveen THA, Ferwerda G, Ahout IM, Vissers M, de Groot R, Boekhorst $J$, et al. Haemophilus is overrepresented in the nasopharynx of infants hospitalized with RSV infection and associated with increased viral load and enhanced mucosal CXCL8 responses. Microbiome. 2018;6(1):10.

46. Clucas DB, Carville KS, Connors C, Currie BJ, Carapetis JR, Andrews RM. Disease burden and health-care clinic attendances for young children in remote Aboriginal communities of northern Australia. Bull World Health Organ. 2008;86(4):275-81.

47. Jacoby P, Carville KS, Hall G, Riley TV, Bowman J, Leach AJ, et al. Crowding and other strong predictors of upper respiratory tract carriage of otitis media-related bacteria in Australian Aboriginal and non-Aboriginal children. Pediatr Infect Dis J. 2011;30(6):480-5.

48. Gunasekera H, Morris PS, Daniels J, Couzos S, Craig JC. Otitis media in Aboriginal children: the discordance between burden of illness and access to services in rural/remote and urban Australia. J Paediatr Child Health. 2009;45(7-8):425-30.

49. DeLacy J, Dune T, Macdonald JJ. The social determinants of otitis media in Aboriginal children in Australia: are we addressing the primary causes? A systematic content review. BMC Public Health. 2020;20(1):492.

\section{Publisher's Note}

Springer Nature remains neutral with regard to jurisdictional claims in published maps and institutional affiliations.

Ready to submit your research? Choose BMC and benefit from:

- fast, convenient online submission

- thorough peer review by experienced researchers in your field

- rapid publication on acceptance

- support for research data, including large and complex data types

- gold Open Access which fosters wider collaboration and increased citations

- maximum visibility for your research: over $100 \mathrm{M}$ website views per year

At BMC, research is always in progress.

Learn more biomedcentral.com/submissions 